\title{
The Effects of Interspecific Courtship on Mating Success in Drosophila melanogaster
}

\author{
Rebecca Dawson \\ Department of Biology, Saint Joseph's University \\ 5600 City Avenue, Philadelphia PA, 19131, USA \\ Scott P. McRobert (Corresponding author) \\ Department of Biology, Saint Joseph's University \\ 5600 City Avenue, Philadelphia PA, 19131, USA \\ E-mail: smcrober@sju.edu
}

Received: August 16, 2011

doi:10.5539/ijps.v3n2p14
Accepted: October 13, $2011 \quad$ Published: December 1, 2011

URL: http://dx.doi.org/10.5539/ijps.v3n2p14

\begin{abstract}
Two sympatric, distantly related Drosophila species, D. melanogaster and D. affinis have been shown to interact sexually. Mature $D$. melanogaster males perform vigorous courtship in response to both virgin and mated $D$. affinis females, but perform no courtship in response to virgin females of another sympatric species, $D$. immigrans. To determine whether inter-specific courtship might interfere with mating success and fitness, $D$. affinis females were used as 'interference sex objects' in tests where D. melanogaster males had access to con-specific females. In these tests the presence of $D$. affinis females was shown to significantly reduce the mating success of $D$. melanogaster males. In comparison, the presence of mated D. melanogaster females or virgin $D$. immigrans females had no impact on the mating success of $D$. melanogaster males in similar tests.
\end{abstract}

Keywords: Drosophila melanogaster, Drosophila affinis, Inter-specific courtship, Mating success

\section{Introduction}

The mating system for Drosophila melanogaster appears to be based on female choice. Females, the discriminating sex, assess male courtship and control whether or not copulation occurs. Males, the indiscriminant sex, approach any fly-like object and, if sexually stimulated, perform a complex series of courtship behaviors and attempt to mate. If the fly that the male approaches is a con-specific female, the male will pursue her and repeat the courtship display until she slows down and opens vaginal plates to allow copulation to occur (reviewed in Greenspan \& Ferveur, 2000). In this way males may demonstrate their fitness as an ability to perform the correct, species-specific courtship display, and females select males who have successfully caused them to become sexually receptive.

From an evolutionary perspective it would seem critical for Drosophila males to avoid directing their courtship toward flies from other species. Inter-specific courtship would appear to be a waste of time and energy and, if copulation ensued, gametes (Spieth \& Ringo, 1983). Furthermore, since courtship subjects flies to an increased predation risk, it would seem likely that mechanisms might evolve that would reduce the amount of courtship males perform toward other species. And, indeed, this appears to be true as in many instances Drosophila males either do not initiate courtship of non-con-specific flies, or quickly terminate courtship of these flies (Spieth, 1974; Spieth \& Ringo, 1983).

There are, however, some instances in which flies engage in inter-specific courtship. One such situation has been reported for interactions between D. melanogaster and D. affinis, the two most common Drosophila species in the Philadelphia area (McRobert \& Tompkins, 1986a). D. melanogaster and D. affinis have been shown to engage in prolonged inter-specific courtship, with males from each species courting members of the other species (McRobert \& Tompkins, 1986b; McRobert \& Tompkins, 1988). Both D. melanogaster and D. affinis encounter each other in the wild, utilizing natural food sources together (McRobert, pers. notes) and both species are attracted to traps baited with bananas and yeast. The male courtship displays of these species are very similar, 
with males from both species orienting toward the female, following her if she moves away, tapping at her with fore-tarsi and producing a courtship song by vibrating their wings (McRobert \& Tompkins, 1986b). In addition, $D$. melanogaster perform a behavior known as 'licking' in which they contact the female with their proboscis. $D$. affinis males don't perform the licking behavior (Miller, 1950). However, despite the similarities in sexual behavior, $D$. melanogaster and $D$. affinis are only distantly related and probably cannot produce viable hybrids since they have different numbers of chromosomes (Wheeler, 1981; Lakovaara \& Saura, 1982).

In this report we examine the possible consequences of inter-specific courtship from the perspective of the $D$. melanogaster male. In short, do D. melanogaster males experience lowered mating success with con-specific females when in the presence of $D$. affinis females? In addition, we addressed the question of whether inter-specific courtship might lower the reproductive fitness of $D$. melanogaster males.

\section{Methods}

\subsection{Stocks}

Fly stocks were derived from fertilized females collected in Rosemont, Pennsylvania in 2003 (D. melanogaster) and 2005 (D. affinis and D. immigrans). All flies were maintained on Carolina Instant Media (Formula 4-24) at $22-26^{\circ} \mathrm{C}$ under a $12: 12 \mathrm{LD}$ cycle. For the $D$. affinis stock, liquefied opuntia cactus was added to the media, which appeared to increase the vitality of the flies.

\subsection{Behavioral Observations}

All behavioral observations were conducted between 8 am $-12 \mathrm{pm}$, at $21-26^{\circ} \mathrm{C}$. Virgin flies were collected under $\mathrm{CO} 2$ anesthesia between $3-12$ hours post-eclosion. Males were housed singly, while females were housed in groups from 2 to 10 in vials containing yeasted media. Flies were tested 3- 5 days post-eclosion.

\subsection{Copulation Tests}

Three separate copulation tests were conducted, including: D. melanogaster males with virgin $D$. melanogaster females, $D$. melanogaster males with mated $D$. melanogaster females, and D. melanogaster males with virgin $D$. affinis females.

For each test a single male was aspirated into a food vial containing a single female and observed for sixty minutes. During the observation period, copulation success or failure, copulation latency (the time until copulation began), and copulation duration (the time the pair stayed in copula) were timed and recorded. In tests involving mated females, the females were tested 24 hours post-mating. No individual fly was tested more than once.

\subsection{Courtship Index Tests}

For each test a single male was aspirated into a Plexiglas chamber (volume $=0.4 \mathrm{~cm}^{3}$ ) along with a sex object and observed for 10 minutes. The courtship index (the fraction of the observation period during which the $D$. melanogaster male performed courtship behaviors) was timed and recorded. Behaviors classified as courtship included orientation, tapping, following, singing, licking and attempted copulation (see Tompkins, 1984; Hall, 1994; McRobert, 2004). If copulation occurred the test was ended and the courtship index was determined for the period of time leading up to copulation. Sex objects included $D$. melanogaster virgin females, mated $D$. melanogaster females, $D$. affinis virgin females, mated $D$. affinis females, and virgin $D$. immigrans females.

\subsection{Courtship Interference Tests}

For each test a single $D$. melanogaster male was aspirated into a food vial containing five "target" $D$. melanogaster virgin females along with nothing (for baseline), or five "interference" sex objects, and observed for ninety minutes. During each test the total number of copulations, copulation latency until the first successful copulation, and copulation duration were timed and recorded. The interference groups included five mated $D$. melanogaster females, five $D$. affinis virgin females, or five virgin $D$. immigrans females.

\subsection{Offspring Production}

In these tests, individual $D$. melanogaster males were mated with individual virgin $D$. melanogaster females in food vials. Following copulation the males were removed from the vials while the mated females remained in order to allow each female to lay eggs. After seven days, each female was transferred to a new food vial. This was performed twice to allow each mated female 21 days to produce eggs. Eggs and larvae were counted using a sucrose extraction technique (Frank, Adams \& McRobert, 1995).

\subsection{Statistical Analysis}

The significance of difference in copulation frequency was determined with a $2 \times 2$ contingency chi-square test. All other comparisons were made using a one-way ANOVA followed by a post-hoc Tukey HSD test. Since courtship indices are percentages, these data were arcs in transformed before being analyzed. 


\section{Results}

Courtship index tests were used to assess the relative degree of attraction of D. melanogaster males to different sex objects. $D$. melanogaster males performed significantly more courtship in response to virgin $D$. melanogaster females than in response to mated D. melanogaster females $(\mathrm{P}<0.001)$, virgin $D$. affinis females $(\mathrm{P}=0.001)$, and virgin $D$. immigrans females $(\mathrm{P}<0.001)$. There was no significant difference in the amount of courtship D. melanogaster males performed toward virgin D. melanogaster females and mated $D$. affinis females $(\mathrm{P}=0.128)$. There was also no significant difference between the amount of courtship performed by $D$. melanogaster males toward virgin $D$. affinis females and mated $D$. affinis females $(\mathrm{P}=0.3419)$. Finally, $D$. melanogaster males performed significantly more courtship in response to either virgin $D$. affinis females or mated D. affinis females than in response to D. immigrans females $(\mathrm{P}<0.001$ and $\mathrm{P}<0.001$ respectively). These results are summarized in Figure I.

Copulation frequency tests were used to determine the probability of mating between D. melanogaster males and different types of females. D. melanogaster males copulated with virgin D. melanogaster females significantly more often than with mated $D$. melanogaster females $(\mathrm{P}<0.001)$ or virgin $D$. affinis females $(\mathrm{P}<0.001)$. $D$. melanogaster males copulated with virgin $D$. affinis females significantly more often than with mated $D$. melanogaster females $(\mathrm{P}=0.003)$. These results are summarized in Figure II.

Courtship interference tests were used to determine whether the presence of certain types of flies ('interference sex objects') affected the mating success of $D$. melanogaster males with virgin $D$. melanogaster females. In these tests the presence of mated $D$. melanogaster females or virgin $D$. immigrans females caused no significant reduction in con-specific matings for $D$. melanogaster males when compared to control tests with no interference sex objects ( $\mathrm{P}=0.9998$ and $\mathrm{P}=0.9970$ respectively). However, the presence of five virgin $D$. affinis females led to a significant reduction in the number of con-specific mating by $D$. melanogaster males when compared to controls $(\mathrm{P}<0.001)$. These results are summarized in Figure III.

\section{Discussion}

As reported in earlier studies, the two most common Drosophila species in the Philadelphia area, $D$. melanogaster and D. affinis (McRobert \& Tompkins, 1986a) interact sexually despite their relatively distant taxonomic relationship and the fact that hybrization between these species does not lead to viable offspring (McRobert \& Tompkins, 1986b; McRobert \& Tompkins, 1988). This raises the question of whether inter-specific courtship between these species might have negative consequences. In this report we show that the presence of $D$. affinis females leads to a reduction in their mating success by D. melanogaster males with con-specific females.

While D. melanogaster males performed the highest level of courtship toward virgin con-specific females, they performed more courtship toward both virgin and mated D. affinis females than toward mated females of their own species. Furthermore, $D$. melanogaster males copulated with virgin $D$. affinis females to a greater degree than with mated $D$. melanogaster females. It has been well established that $D$. melanogaster males provide females with a seminal-fluid compound known as sex peptide during copulation (Chapman, 2001). Sex peptide acts within the body of the recently mated female to reduce her production of aphrodisiac pheromone and reduce her receptivity to copulation for a period of a few days (Chen, Stumm-Zollinger, Aigaki, Balmer, Bienz, \& Bohlen, 1988; Chapman, Bangham, Vinti, Seifried, Lung, Wolfner, Smith \& Partridge, 2003). In this way, recently mated $D$. melanogaster females stimulate less courtship from males and are less likely to re-mate during the period of time when they are laying eggs. This system protects the sperm of the male that mated with the female, but also acts to reduce the time that other males spend courting a female who is unlikely to copulate. However, despite the fact that inter-specific matings between $D$. melanogaster and $D$. affinis never result in the production of viable offspring, no mechanism appears present to reduce the inter-specific courtship observed between these species.

The negative effects of inter-specific courtship between $D$. melanogaster and $D$. affinis were evident in interference tests. When placed into a scenario with five con-specific virgin females and either five mated $D$. melanogaster females or five virgin $D$. immigrans females (a sympatric species that does not elicit courtship from D. melanogaster males), D. melanogaster males copulated with an average of three of the con-specific virgins. This number was equivalent to the average number of copulations that occurred in the control scenario with no interference flies. These results suggest that the presence of either con-specific, mated females or virgin D. immigrans females had no effect on intra-specific courtship and copulation in D. melanogaster. However, the presence of five $D$. affinis females significantly reduced the number of copulations, indicating that inter-specific courtship between $D$. melanogaster and $D$. affinis had a detrimental impact on courtship and mating in $D$. melanogaster males. 
To address the idea of how the loss of mating success might affect fitness in D. melanogaster males, an offspring production analysis was performed. In the laboratory, D. melanogaster females produced an average of 443.8 $(+/-25.1)$ offspring from a single mating. Adding this information to the results of the interference tests, mating with three females (as males did in assays that did not include $D$. affinis females) would lead to the production of approximately 1331.4 offspring. In assays that included D. affinis females, males mated with an average of 2.17 females, which would lead to a production of approximately 963 offspring, a reduction of 368 offspring per session. Obviously, these numbers relate to the carefully controlled atmosphere of the laboratory, but still illustrate the point that the presence of $D$. affinis females could lead to lowered offspring production in $D$. melanogaster males.

From a traditional point of view, elaborate courtship displays prior to copulation evolve as reproductive isolation mechanisms, preventing the production of hybrids with reduced fitness (reviewed in Thornhill \& Adcock, 1983). Selection would thus favor individuals that avoid the fitness costs associated with hybridization. However, due to the costs of courtship (time, energy and the risk of predation), selection should also favor animals that restrict the prolonged courtship of non-con-specifics. For example, males from the two sibling species, $D$. affinis and $D$. algonquin, do not initiate courtship or attempt copulation with non-con-specific females when placed in mixed-species groups (Miller, 1950). Similarly, D. melanogaster males perform little or no courtship toward two sibling species, D. simulans and D. mauritiana (Manning, 1959; Schilder \& Dow, 1977; Robertson, 1983). In this study, D. melanogaster males performed no courtship in response to $D$. immigrans females, which are sympatric with D. melanogaster in the Philadelphia area.

Not all species have such firm barriers against sexual attraction and interaction, however. For example, $D$. persimilis and D. pseudoobscura, which overlap in territory in the western United States, will court each other and produce fertile hybrid offspring in the lab even though hybrids are rarely seen in nature (Mayr, 1946). In the closely related sibling species $D$. melanogaster and $D$. simulans, which have strong sexual isolation in nature but court in the laboratory setting, interbreeding leads to the production of sterile hybrids (Barker, 1962), and $D$. melanogaster males even prefer courting $D$. simulans females to con-specific females, though the acceptance of D. simulans females is quite low (Wood \& Ringo, 1980).

For species that interact sexually, learning may act to reduce interactions that waste time and energy and do not lead to the production of viable hybrids. In a phenomenon known as experience-dependent courtship modification, D. melanogaster males reduce their courtship toward mated or virgin con-specific females following courtship of a mated female (Gailey, Jackson \& Siegel, 1982; Seigel \& Hall, 1979). Similar mechanisms may also be involved in inter-specific situations. For instance, in interactions between $D$. persimilis and D. pseudoobscura, D. persimilis males who had experienced courtship and rejection by D. pseudoobscura females consequently exhibited lowered levels of hetero-specific courtship compared to inexperienced males (Dukas, 2008). Similarly, Dukas (2004) showed that D. melanogaster males previously exposed to D. simulans females showed significantly reduced levels of courtship towards $D$. simulans females but maintained normal levels of courtship towards D. melanogaster females. Expanding this to a molecular level, Ellis and Carney (2009) used microarray analysis to identify altered expression of nine genes in D. melanogaster males that had courted D. simulans females. In contrast, thirty-five genes displayed altered expression in D. melanogaster males that had courted con-specific females. Such alterations in gene expression may represent a system that both reduces hetero-specific courtship and encourages con-specific courtship. It is possible that a similar mechanism may function to lessen interactions between $D$. melanogaster and $D$. affinis although, within the ninety minute periods utilized in our courtship interference tests, the presence of $D$. affinis females still apparently led to a reduction in con-specific mating success for the D. melanogaster males.

Finally, it is difficult to use laboratory studies to make definitive statements about reproductive isolation and fitness in natural populations. While the results presented here suggest that the presence of $D$. affinis may negatively impact mating success and fitness in D. melanogaster, other factors may occur in the wild that dramatically affect the interactions between species. However, laboratory studies like these offer an intriguing look at the effects of one species on another and provide a foundation for further work. In future two other aspects of $D$. melanogaster- D. affinis interaction will be addressed: First, laboratory tests will be performed to determine whether inter-specific courtship leads to courtship modification in D. melanogaster males. If true, $D$. melanogaster males may learn to reduce the time they spend courting $D$. affinis females. Second, field studies will be performed in an attempt to provide a detailed description of inter-specific courtship between $D$. melanogaster and D. affinis in the wild. 


\section{Acknowledgments}

We thank Kate Buehler for help with the maintenance and testing of the flies. This project was supported, in part, by a grant from the Saint Joseph's University Sigma Xi Chapter.

\section{References}

Amrein, H. (2004). Pheromone perception and behavior in Drosophila. Curr. Opin. Neurobiol, 14: $435-442$. http://dx.doi.org/10.1016/j.conb.2004.07.008

Barker, J.S.F. (1962). Sexual isolation between Drosophila melanogaster and Drosophila simulans. Am. Nat, 96(887): 105-115. http://dx.doi.org/10.1086/282211

Chapman, T. (2001). Seminal fluid-mediated fitness traits in Drosophila. Heredity , 87: 511-521. http://dx.doi.org/10.1046/j.1365-2540.2001.00961.x

Partridge, L. (2003). The sex peptide of Drosophila melanogaster: Female post-mating responses analyzed by using RNA interference. Proc. Natl. Acad. Sci. USA 100: 9923-9928. http://dx.doi.org/10.1073/pnas.1631635100

Chen, P. S., Stumm-Zollinger, E., Aigaki, T. Balmer, J., Bienz, M., \& Bohlen, P. (1988). A male accessory gland peptide that regulates reproductive behavior of female D. melanogaster. Cell, 54: 291-298, http://dx.doi.org/10.1016/0092-8674(88)90192-4

Dukas, R. (2004). Male fruit flies learn to avoid interspecific courtship. Behav. Ecol, 15(4): 695-698, http://dx.doi.org/10.1093/beheco/arh068

Dukas, R. (2008). Learning decreases heterospecific courtship and mating in fruit flies. Biol. Letters 4: 645-647. http://dx.doi.org/10.1098/rsbl.2008.0437

Ellis, L.L., \& Carney, G.E. (2009). Drosophila melanogaster males respond differently at the behavioural and genome-wide levels to Drosophila melanogaster and Drosophila simulans females. J. Evol. Biol, 22: $2183-2191$. http://dx.doi.org/10.1111/j.1420-9101.2009.01834.x

Frank, J. Adams, C., \& McRobert, S.P. (1995). Sucrose density separation of Drosophila larvae from instant medium. Dros. Info. Serv, 76:172

Gailey, D.A. Jackson, \& F.R. Siegel, R.W. (1982). Male courtship in Drosophila: The conditioned response to immature males and its genetic control. Genetics, 102: 771-782

Greenspan, R.J. Ferveur, J. (2000). Courtship in Drosophila. Annu. Rev. Genet, 34: $205-232$. http://dx.doi.org/10.1146/annurev.genet.34.1.205

Hall, J.C. (1994). The mating of a fly. Science 264: 1702-1714. http://dx.doi.org/10.1126/science.8209251

Lakovaara, S., \& Saura, A. (1982). Evolution and speciation in the Drosophila obscura subgroup. In M. Ashburner, H.L. Carson, and J.N. Thompson (eds.), The Genetics and Biology of Drosophila, Vol. 3b. Academic Press, New York, 1-59

Manning, A. (1959). The sexual behavior of two sibling Drosophila species. Behaviour 15: $123-145$. http://dx.doi.org/10.1163/156853960X00133

Mayr, E. (1946). Experiments on sexual isolation in Drosophila. VII. The nature of the isolating mechanisms between D. pseudoobscura and D. persimilis. Proc. Natl. Acad. Sci. USA 32 (5): $128-137$. http://dx.doi.org/10.1073/pnas.32.5.128

McRobert, S.P., \& Tompkins, L. (1986a). Drosophila species in the Philadelphia area. Dros. Info. Serv. $63: 95$.

McRobert, S.P., \& Tompkins, L. (1986b). Incomplete behavioral isolation between two distinctly related Drosophila species. Evolution, 40(6): 1185-1190. [Online] Available: http://www.jstor.org/stable/2408946

McRobert, S.P., \& Tompkins, L. (1988). Two consequences of homosexual courtship performed by Drosophila melanogaster and Drosophila affinis males. Evolution 42(5): 1093-1097. [Online] Available: http://www.jstor.org/stable/2408925

McRobert, S.P. (2004). Reproductive behavior: Sexual behavior in fruit flies - Drosophila. Encyclopedia of Animal Behavior, 930-934

Miller, D.D. (1950). Mating behavior of Drosophila affinis and Drosophila algonquin. Evolution, 4: $123-134$. http://dx.doi.org/10.2307/2405389 
Robertson, H.M. (1983). Mating behavior and the evolution of Drosophila mauritiana. Evolution, 37: 1283-1293. http://dx.doi.org/10.2307/2408848

Schilder, F. Dow, M. (1977). Courtship behavior in Drosophila: Sexual isolation or sexual selection? Z. Tierpsychol, 43: 304-310. http://dx.doi.org/10.1111/j.1439-0310.1977.tb00077.x

Siegel, R.W. Hall, J.C. (1979). Conditioned responses in courtship behavior of normal and mutant Drosophila. Proc. Natl. Acad. Sci. USA 76(7): 3430-3434. http://dx.doi.org/10.1073/pnas.76.7.3430

Singh, S.R. Singh, \& B.N. Hoenigsberg, H.F. (2002). Female remating, sperm competition and sexual selection in Drosophila. Genet. Mol. Res, 1(3): 178-215

Spieth, H.T. (1974). Courtship behavior in Drosophila. Annu. Rev. Entomol, 19: 383-406, http://dx.doi.org/10.1146/annurev.en.19.010174.002125

Spieth, H.T., \& Ringo, J.M. (1983). Mating behavior and sexual isolation in Drosophila. In M. Ashburner, H.L. Carson, and J.N. Thompson (eds.), The Genetics and Biology of Drosophila, Vol. 2c. Academic Press, New York, pp. 224-284.

Thornhill, R., \& Alcock, J. (1983). The evolution of insect mating systems. Harvard University Press, Cambridge, Massachusetts.

Tompkins, L. (1984). Genetic analysis of sex appeal in Drosophila. Behav. Genet, 14: 411-440, http://dx.doi.org/10.1007/BF01065443

Wheeler, M.R. (1981). The Drosophilidae: A taxonomic overview. In M. Ashburner, H.L. Carson \& J.N. Thompson (Eds.). The Genetics and Biology of Drosophila, 3, 1-97. New York: Academic Press.

Wood, D., \& Ringo, J.M. (1980). Male mating discrimination in Drosophila melanogaster, D. simulans and their hybrids. Evolution, 34(2): 320-329. [Online] Available: http://www.jstor.org/stable/2407395

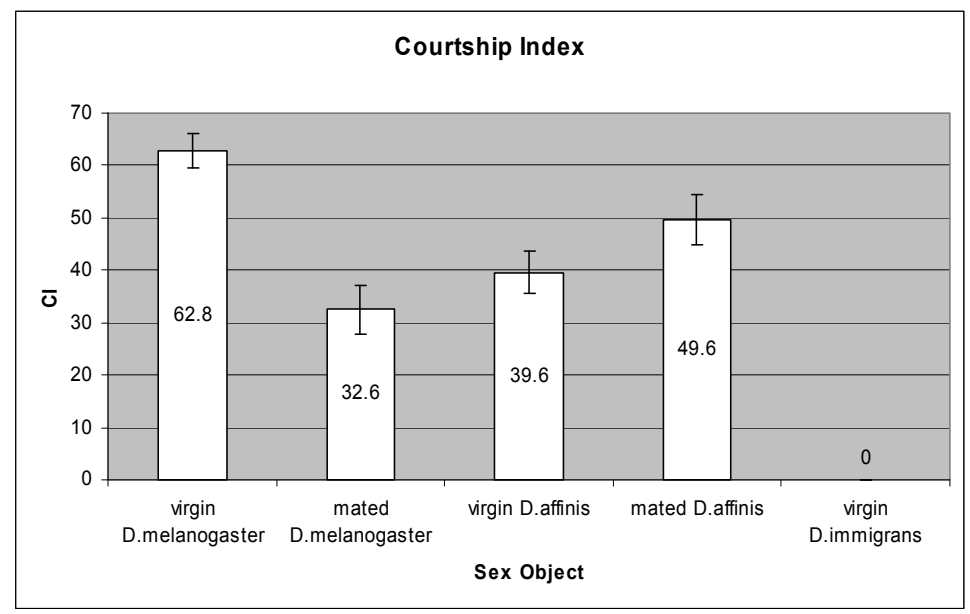

Figure 1. The courtship index of D. melanogaster males in response to different sex objects

$\mathrm{N}=20$ for each bar 


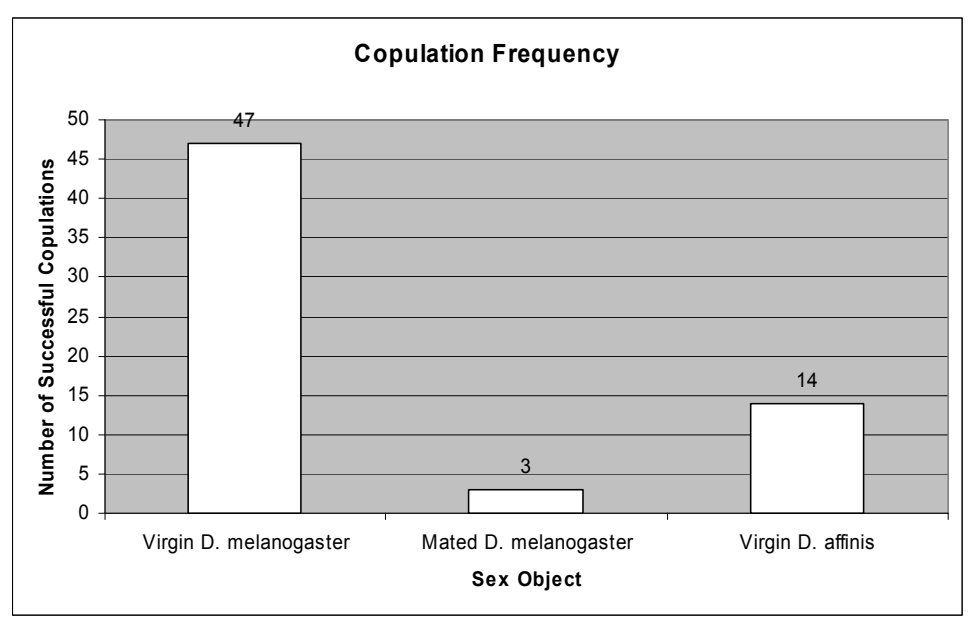

Figure 2. The copulation frequency of $D$. melanogaster males with different sex objects.

$\mathrm{N}=50$ for each bar.

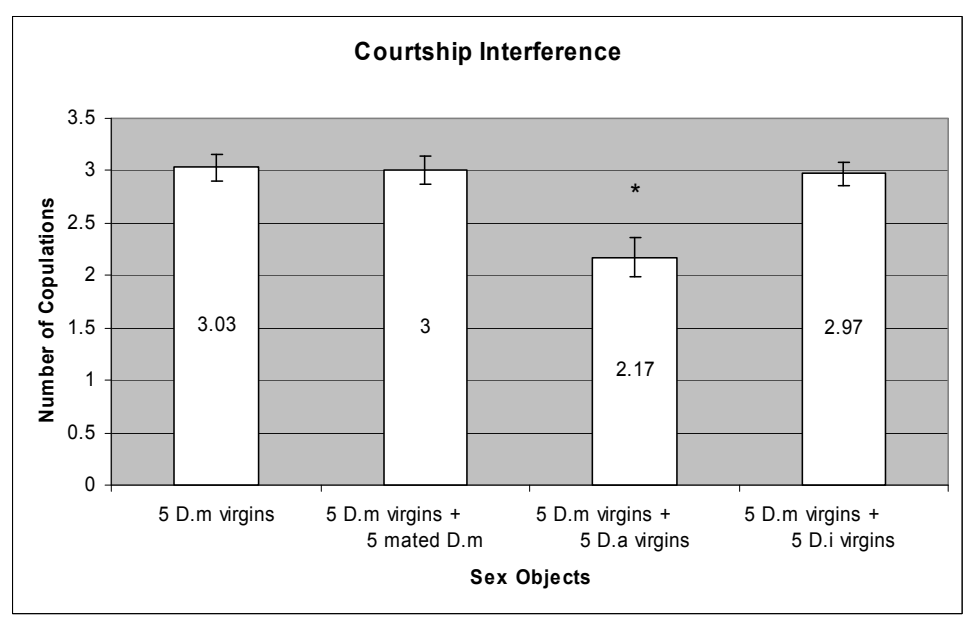

Figure 3. The effects of different "interference sex objects" on the mating success of D. melanogaster males with five conspecific virgin females. $\mathrm{N}=30$ for each bar. * denotes the group that was significantly different from the rest 